\title{
Zeeman NQR Studies on Powdered Bromine Compounds Having Small $\eta^{*}$
}

\author{
Osamu Ege \\ Department of Physics, Faculty of Education, Miyazaki University, Gakuen-kibanadai, \\ Miyazaki 889-21, Japan \\ Hisao Negita \\ Computer Center, Hiroshima University of Economics, Gion, Asaminami, Hiroshima 731-01, Japan
}

Z. Naturforsch. 45a, 599-602 (1990); received August 26, 1989; in revised form November 24, 1989

The Zeeman NQR of ${ }^{79} \mathrm{Br}$ and ${ }^{81} \mathrm{Br}$ in samples having relatively small $\eta$ was studied on several bromine compounds which were in the powder or polycrystal state. The absorption lines were detected in the integral form by the bridge circuit. When a static magnetic field was applied, the line shapes were broadened and usually two dips appeared symmetrically at both sides of the zero field line. The values of $\eta$ were estimated from the patterns of the dips. For compounds such as $\mathrm{NaBrO}_{3}$, however, as expected no dips were observed.

\section{Introduction}

Powder Zeeman NQR (PZNQR) was theoretically studied by Toyama and Morino [1, 2]. Brooker and Creel developed it by computer simulation [3]. Graybeal and Green studied it experimentally [4]. We have also studied and observed PZNQR on $\mathrm{Br}$ and $\mathrm{Cl}$ in several compounds $[5,6]$.

In the case of nuclear spin $I=3 / 2$, the asymmetry parameter $(\eta)$ of the electric field gradient can not be determined only from the measurements of NQR frequencies. Accordingly, it is usually derived from the Zeeman pattern of single crystal samples. Otherwise, it can be approximately estimated from the patterns of PZNQR. This method is useful when single crystals are not easy to prepare.

The patterns of PZNQR are grouped into the following three types $[2,3,5,6]$. Type 1 : the pattern has two clear dips at both sides of the original line (zero field line). In this type, the value of $\eta$ is estimated from the geometry of the dips and gives a larger value than about 0.05 . Type 2 : the pattern yields two slight and broad mounds at both sides of the original line. In this case, $\eta$ is estimated to be smaller than about 0.05 , but not zero. Type 3: when there is a broad and arc

\footnotetext{
* Presented at the Xth International Symposium on Nuclear Quadrupole Resonance Spectroscopy, Takayama, Japan, August 22-26, 1989.

Reprint requests to Dr. O. Ege, Department of Physics, Faculty of Education, Miyazaki University, Gakuen-kibanadai, Miyazaki 889-21, Japan.
}

like mound at the middle and two shoulders at both sides of it, $\eta$ is expected to be very small. In any case it should be noted that $\eta$ estimated by the method of PZNQR might have an uncertainty of around 0.05 . This results from the circumstance that the frequency of the NQR oscillator gradually shifts during the data accumulation in the signal averager and the pattern is widely deformed. Therefore, the accumulating time must be as short as possible.

\section{Experimental}

The PZNQR due to $\mathrm{Br}$ nuclei in several compounds was studied at liquid $\mathrm{N}_{2}$ (LN) temperature using a modified Schawlow's type NQR oscillator. As shown in Fig. 1, the NQR oscillating coil was kept parallel to the Helmholtz-coil being able to supply static magnetic fields to the samples. A Dewar vessel filled with LN was set to cool the samples. The NQR signals were detected by the bridge circuit in the integral form and led to a signal averager to enhance the $\mathrm{S} / \mathrm{N}$ $[5,6,7]$.

With a frequency modulation NQR oscillator operated at a low sweep speed, the signals were accumulated by the averager, and the patterns of the PZNQR were recorded. A saw-tooth voltage was supplied on a varicap-diode which generated the frequency modulation synchronizing for the accumulation. If the modulating frequency is perfectly linear with respect to the supplied voltage on the diode, $\eta$ can be estimated from 


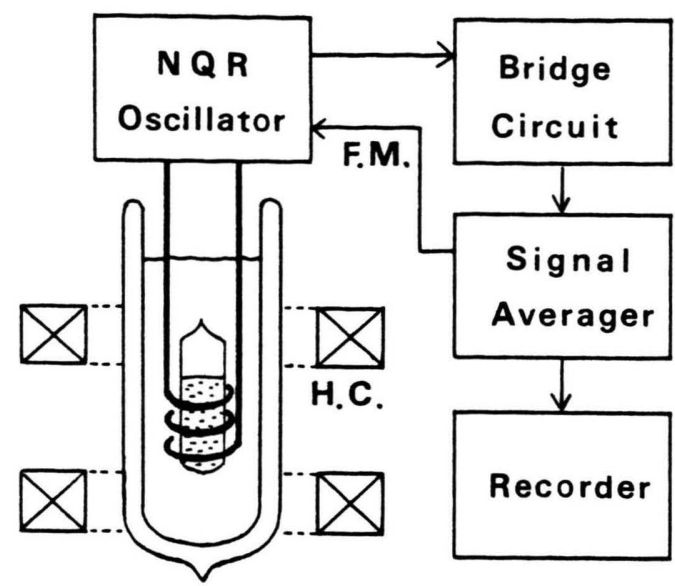

1

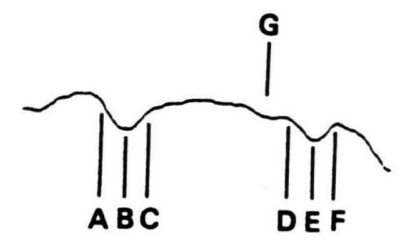

3

Fig. 1. Block diagram of the arrangement for the PZNQR. F.M. is the frequency modulation and H.C. the Helmholtz-coil.

Fig. 3. $P Z N Q R$ pattern of $\mathrm{BBr}_{3}$ with two clear dips and its geometry points.

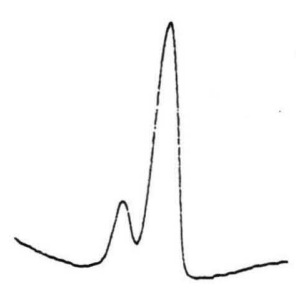

$0 G$

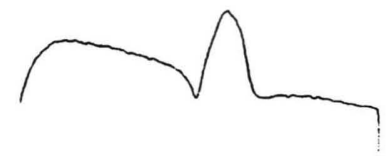

3. $1 \mathrm{G}$
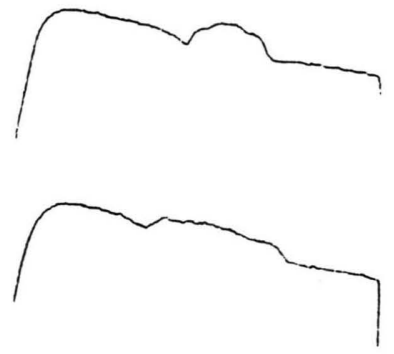

$6.5 \mathrm{G}$

$9.7 \mathrm{G}$

11. $4 G$

$14.6 G$ 2

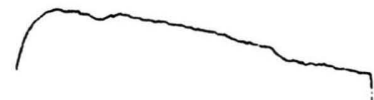

$12.9 G$ 4

Fig. 4. PZNQR patterns due to ${ }^{79} \mathrm{Br}$ in $\mathrm{NaBrO}_{3}$.

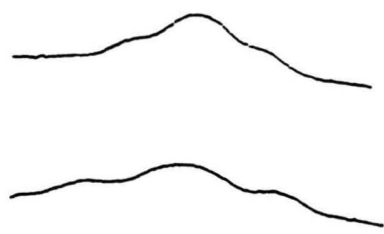

$12.7 G$

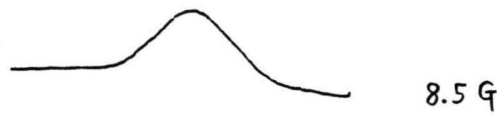

$17.1 \mathrm{G}$

$9.7 G$
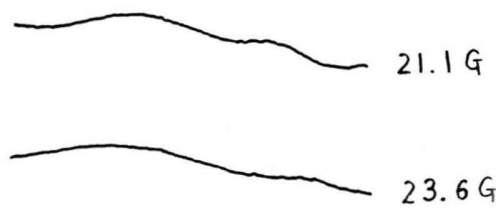
5

Fig. 5. PZNQR patterns due to ${ }^{79} \mathrm{Br}$ in bromobenzene.
Fig. 2. PZNQR patterns due
${ }^{79} \mathrm{Br}$ in $\mathrm{BBr}_{3}$ at increasing field. 

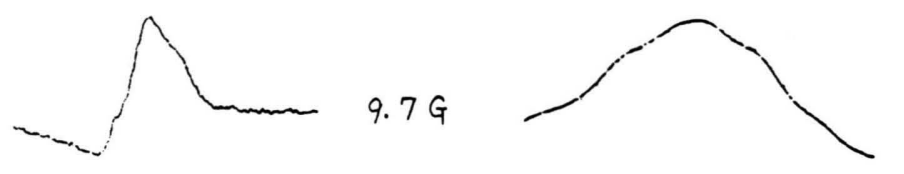

$17.9 \mathrm{G}$
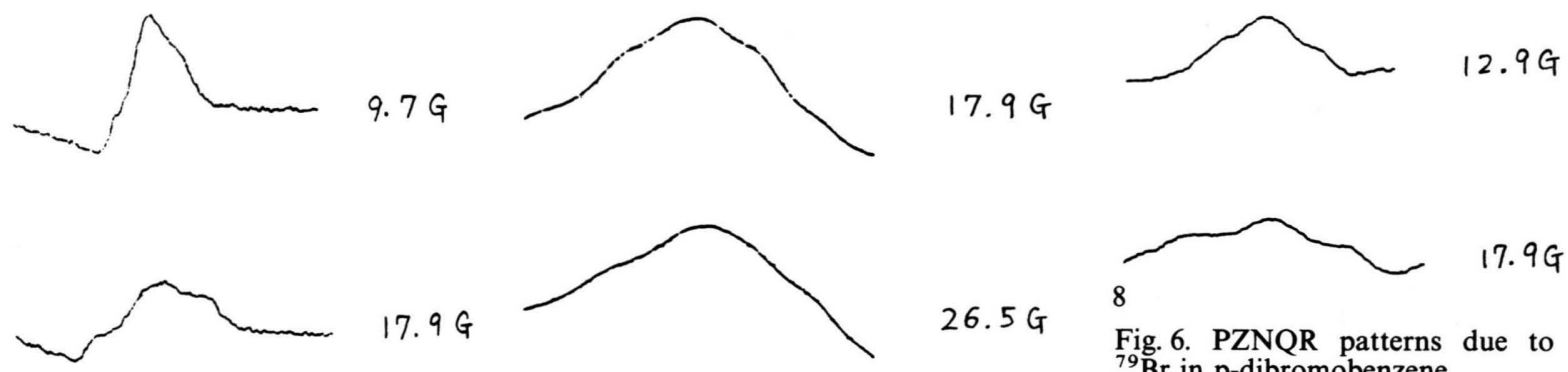

$17.9 G$

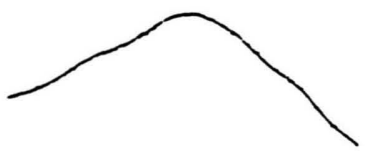

$26.5 \mathrm{G}$

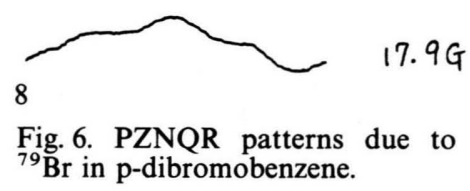

Fig. 7. $P Z N Q R$ patterns due to ${ }^{81} \mathrm{Br}$ in $\mathrm{C}_{2} \mathrm{H}_{5} \mathrm{Br}$.

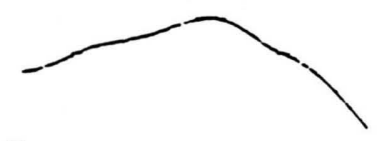

35. $O G$

Fig. 8. PZNQR patterns due to 7

the geometrical points (type 1) or the figures (type 2 and 3) of the patterns recorded [6]. The above linear condition was also sufficiently valid in this experiment.

\section{Results and Discussion}

$\mathrm{BBr}_{3}$ is liquid at room temperature. Its $\mathrm{PZNQR}$ pattern due to ${ }^{79} \mathrm{Br}$ was detected with increasing the static magnetic field. The result is shown in Figure 2.

There are two resonance absorption lines originally (one line two times stronger than the other). The stronger line has the frequnecy $175.298 \mathrm{MHz}$. The weaker of $175.273 \mathrm{MHz}$ is broadened and buried in the pattern of the stronger one with increasing the field.

As shown in Fig. 3, the pattern has two clear dips (type 1), and $\eta$ is estimated by the following expression (see [5]):

$$
\begin{aligned}
\eta & =\frac{\left(v_{C}-v_{A}\right)+\left(v_{F}-v_{D}\right)}{2\left(v_{E}-v_{B}\right)} \\
& =\frac{\overline{A C}+\overline{D F}}{2 \overline{B E}},
\end{aligned}
$$

where $v_{A}, v_{B}, \ldots, v_{F}$ are the frequencies at the points $A, B, \ldots, F$, and $\overline{A C}, \overline{D F}$ and $\overline{B E}$ are the horizontal distances between the points $A, B, \ldots, F$ of the PZNQR curve recorded. The $\eta$ value estimated from a number of patterns is 0.26 . This is comparatively smaller than the value 0.45 by Chiba [8], which was determined by use of the single crystal method. We can see part of the shoulder of the lower frequency at
Table 1. $\eta$ and the other constants of ${ }^{79} \mathrm{Br}$ and ${ }^{81} \mathrm{Br}$ in several compounds.

\begin{tabular}{lllll}
\hline $\begin{array}{l}\text { Com- } \\
\text { pounds }\end{array}$ & Nuclei & $\begin{array}{l}\text { Frequency } \\
\text { (MHz) }\end{array}$ & $\eta$ & $\begin{array}{l}e^{2} q Q h^{-1} \\
(\mathrm{MHz})\end{array}$ \\
\hline $\mathrm{BBr}_{3}$ & ${ }^{79} \mathrm{Br}$ & 175.298 & 0.26 & 346.71 \\
$\mathrm{NaBrO}_{3}$ & ${ }^{79} \mathrm{Br}$ & 181.843 & very small & 363.69 \\
$\begin{array}{l}\text { Bromo- } \\
\text { benzene }\end{array}$ & ${ }^{79} \mathrm{Br}$ & 269.136 & very small & 538.27 \\
$\begin{array}{l}\text { p-Dibromo- } \\
\text { benzene }\end{array}$ & ${ }^{79} \mathrm{Br}$ & 271.119 & very small & 542.24 \\
$\mathrm{C}_{2} \mathrm{H}_{5} \mathrm{Br}$ & ${ }^{81} \mathrm{Br}$ & 207.796 & very small & 415.59 \\
$\mathrm{CH}_{2} \mathrm{Br}$ & ${ }^{81} \mathrm{Br}$ & 234.795 & very small & 469.59 \\
$\mathrm{Br}_{2}$ & ${ }^{81} \mathrm{Br}$ & 319.524 & small & 639.05 \\
$\begin{array}{l}1,4-\mathrm{Dibromo}- \\
\text { butane }\end{array}$ & ${ }^{79} \mathrm{Br}$ & 250.854 & small & 501.71 \\
\hline
\end{tabular}

$G$. As it is closer to the form of the type 3, the value of $\eta$ of the lower frequency might be very small.

The ${ }^{79} \mathrm{Br}$ PZNQR in $\mathrm{NaBrO}_{3}$ was measured with increasing the field. The results are shown in Figure 4. Its signals became broad and characteristic of the type 3 feature, so that $\eta$ was considered to be very small (see [3]).

The ${ }^{79} \mathrm{Br}$ PZNQR of bromobenzene and of p-dibromobenzene and ${ }^{81} \mathrm{Br}$ of $\mathrm{C}_{2} \mathrm{H}_{5} \mathrm{Br}$ and of $\mathrm{CH}_{2} \mathrm{Br}_{2}$ were obtained and are shown in Figs. 5, 6, 7, and 8, respectively. Their $\eta$ 's are also thought to be very small. The $\eta$ of p-dibromobenzene is reported to be smaller than 0.05 by Shimomura [9].

The ${ }^{81} \mathrm{Br}$ PZNQR of $\mathrm{Br}_{2}$ is shown in Figure 9. With increasing field, its pattern had two broad mounds at both sides of the original line. It is thought that the two peaks are overlapping at $\mathrm{H}$ and $\mathrm{I}$ and the very narrow dips are hidden by them. Therefore, it is thought to be in the category of the type 2 and its $\eta$ is 


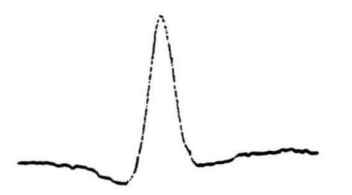

$O G$

$17.9 \mathrm{G}$

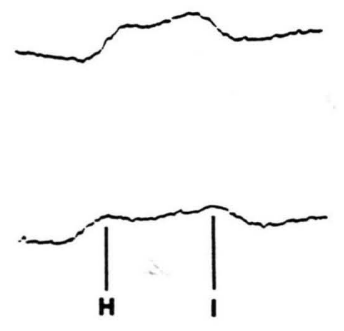

9

$26.5 \mathrm{G}$
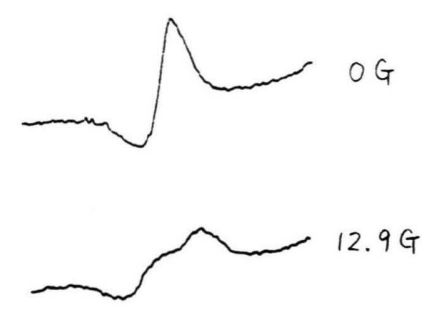

$17.9 \mathrm{G}$
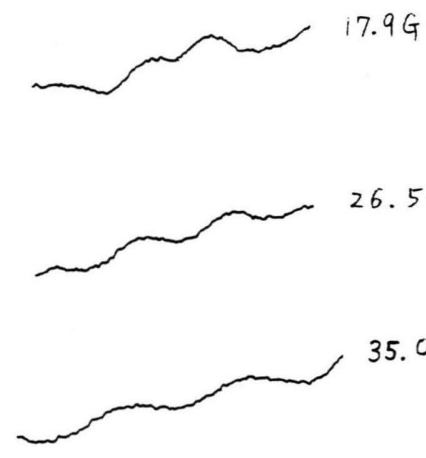

$35.0 \mathrm{G}$

10
Fig. 9. PZNQR patterns due to ${ }^{81} \mathrm{Br}$ in $\mathrm{Br}_{2}$.

Fig. 10. PZNQR patterns due to ${ }^{79} \mathrm{Br}$ in 1,4-dibromobutane. expected to be small, but not zero. In this case, $\eta$ is thought to be smaller than the value 0.2 determined at $-20^{\circ} \mathrm{C}$ by Kojima et al. [10].

The ${ }^{79} \mathrm{Br}$ PZNQR pattern of 1,4-dibromobutane was similarly obtained with the form of type 2 , as shown in Fig. 10, and its $\eta$ is thought to be small.
For these compounds frequencies and the coupling constants determined by assuming $\eta=0$ except for $\mathrm{BBr}_{3}$ at $\mathrm{LN}$ temperature are summarized in Table 1. This method is advantageous for determining whether $\eta$ is small or very small.
[1] M. Toyama, J. Phys. Soc. Japan 14, 1727 (1959).

[2] Y. Morino and M. Toyama, J. Chem. Phys. 35, 1289 (1961).

[3] H. R. Brooker and R. B. Creel, J. Chem. Phys. 61, 3658 (1974).

[4] J. D. Graybeal and P. J. Green, J. Phys. Chem. 73, 2948 (1969).

[5] O. Ege, J. Sci. Hiroshima Univ. A 46, 21 (1982).
[6] O. Ege and H. Negita, the report presented at the IXISNQR (1988, India).

[7] O. Ege, H. Goya, and H. Negita, J. Sci. Hiroshima Univ. A 44, 263 (1980).

[8] T. Chiba, J. Phys. Soc. Japan 13, 860 (1958).

[9] K. Shimomura, J. Phys. Soc. Japan 14, 235 (1959).

[10] S. Kojima, K. Tsukada, A. Shimauchi, and Y. Hinaga, J. Phys. Soc. Japan 9, 795 (1954). 